\title{
Stem Cell Organoids in Primary Cultures of Human Non-Malignant and Malignant Colon
}

Sahrish Tariq 1 , Muhammad Tahseen ${ }^{1}$, Mariam Hassan², Muhammad Adnan Masood ${ }^{3}$, Shahid Khattak ${ }^{4}$, Aamir Ali Syed ${ }^{4}$, Asad Hayat Ahmad', Mudassar Hussain ${ }^{1}$, Muhammed Aasim Yusuf ${ }^{3}$, Chris Sutton ${ }^{5}$, and Saira Saleem ${ }^{6 *}$

${ }^{1}$ Department of Pathology, SKMCH\&RC, 7-A Block R-3, Johar Town, Pakistan

${ }^{2}$ Department of Clinical Research, SKMCH\&RC, 7-A Block R-3, Johar Town, Pakistan

${ }^{3}$ Department of Internal Medicine, SKMCH\&RC, 7-A Block R-3, Johar Town, Pakistan

${ }^{4}$ Department of Surgical Oncology, SKMCH\&RC, 7-A Block R-3, Johar Town, Pakistan

5 Institute of Cancer Therapeutics, University of Bradford, Tumbling Hill Street Bradford, UK

${ }^{6}$ Basic Science Research, SKMCH\&RC, 7-A Block R-3, Johar Town, Pakistan

\begin{abstract}
Aims: A sub-population of cells named cancer stem cells (CSCs) that initiate and promote tumour growth have been demonstrated to exist in several malignancies including colon carcinoma. The objective of our pilot study was to isolate $\mathrm{CD} 133^{+} \mathrm{CD} 26^{+} \mathrm{CD} 44^{+} \mathrm{CSC}$ from patient colon tumours, culture spheres or organoids and observe their proliferation in primary cultures. Parallel cultures of non-cancer controls from colon normal lining and nonadenomatous polyps were set up.
\end{abstract}

Methods: Magnetic activated cell sorting was used to isolate $\mathrm{CD} 133^{+} \mathrm{CD} 26^{+} \mathrm{CD} 44^{+}$cell populations followed by primary cell culturing under stem cell culture conditions. Number, cells/organoid and daughter generations of organoids were calculated using phase contrast microscope. Trypan blue exclusion method was used to test the viability of the cells.

Results: Both colon tumour and colon non-adenomatous polyp formed floating organoids in suspension; however non-adenomatous polyp cultures did not show self-renewal properties for more than 1 passage. Normal colon singlecell suspension did not create organoids. Metastatic colon tumours rapidly produce cancer cell organoids in less than 24 hours in larger numbers compared to non-metastatic colon tumours (1-3 weeks). Metastatic colon tumour organoids have the ability for proliferation for upto five daughter generations in primary culture compared to three generations for those grown from non-metastatic tumours.

Conclusions: This in vitro CSC organoid model will help study colon cancer biology, in particular providing a valuable source of primary cell-derived tissue for studying personalized molecular profiling using 'omics strategies to direct therapeutic intervention.

Keywords: Colon cancer; Primary culture; Cancer cell sphere; Cancer stem cells; In vitro assay

\section{Statement}

This study was performed on primary cultures of fresh sitespecific colon tissues to generate in vitro cancer cell organoid models. As opposed to current literature, we cultured non-cancer colon and compared proliferation patterns to that of tumor tissues and observed differences. This study established a model for in vitro colon tissue generation to study colorectal disease progression.

\section{Introduction}

Colorectal cancer (CRC) is the $3^{\text {rd }}$ most common cancer type worldwide and the $4^{\text {th }}$ most common cause of cancer-related mortality with almost 694,000 people dying annually [1]. According to GloboCan 2012 estimates, colorectal malignancies constituted $10.0 \%$ and $9.2 \%$ of all cancer cases in men and women, respectively. More than half of these cases occurred in developed countries where mortality was substantially lower than the incidence rate. However, more CRC related deaths (52\% in 2012) occurred in countries scoring low on the development index, reflecting relative poor survival in these regions [2]. It has been estimated that there were 95,270 new cases of colon cancer diagnosed in USA and $90 \%$ to $92 \%$ of patients were treated surgically [3-5]. Colorectal cancer incidence and death rates declined by approximately $3 \%$ per year in both men and women from 2003 2012, with momentum gained in the most recent years likely driven by the increased uptake of colonoscopy screening [5]. In Asia, the incidence of colorectal carcinoma is similar but lower than found in the developed countries. The risk of developing the disease is alike in both sexes at present, however a $41 \%$ rise in incidence was noted in the males between 1995-1999 [6]. In a single-center retrospective study of adult patients with colon cancer diagnosed through 2000-2003 (93 patients; $\mathrm{M}: \mathrm{F}=1.58: 1$, mean age of diagnosis 54 years), $49.5 \%$ of the patients had right sided (mortality rate $51.6 \%$ ), $10.8 \%$ had transverse colon (mortality rate $37.5 \%$ ), $7.5 \%$ had descending colon (mortality rate $66.7 \%$ ) and $32.2 \%$ had sigmoid colon (mortality rate $40.9 \%$ ) cancers [7]. At Shaukat Khanum Memorial Cancer Hospital and Research Centre (SKMCH\&RC, Pakistan), CRCs were among the top five malignancies (3530 cases, $4.91 \%$ ) diagnosed amongst all age-groups, both sexes combined registered from 1994 to 2015.

Poor survival rate is in part attributed to limited understanding

*Corresponding author: Saira Saleem, Basic Science Research, Shauka Khanum Memorial Cancer Hospital and Research Centre (SKMCH\&RC), 7-A Block R-3, Johar Town, Lahore, 54000, Pakistan, Tel: +924235905000 Ext. 4367; Fax: +924235945209; E-mail: sairas@skm.org.pk

Received February 17, 2017; Accepted May 26, 2017; Published May 28, 2017

Citation: Tariq S, Tahseen M, Hassan M, Masood MA, Khattak S, et al. (2017) Stem Cell Organoids in Primary Cultures of Human Non-Malignant and Malignant Colon. J Mol Biomark Diagn 8: 346. doi: 10.4172/2155-9929.1000346

Copyright: (c) 2017 Tariq S, et al. This is an open-access article distributed under the terms of the Creative Commons Attribution License, which permits unrestricted use, distribution, and reproduction in any medium, provided the original author and source are credited. 
of the characteristics of resistant cancer cells which are now termed cancer stem cells (CSCs) or tumour initiating cells (TICs). The evidence for the existence of CSCs has been reported in blood borne cancer $\left(\mathrm{CD} 34^{+} \mathrm{CD} 38^{-}\right)[8]$, in solid tumour including breast $\left(\mathrm{CD} 44^{+} \mathrm{CD} 24^{-}\right)$ $[9,10]$, brain $\left(\mathrm{CD} 133^{+}\right)$[11], prostate $\left(\mathrm{CD} 44^{+}\right)$[12] and pancreatic $\left(\mathrm{CD} 44^{+} \mathrm{CD} 24^{+} \mathrm{ESA}^{+}\right)$[13]. The first report on colon cancer stem cells showed that tumourigenic cells were contained within in the rare undifferentiated population that express CD133 [14]. A surface marker profile for isolated CRC stem cells was reported to be epithelial cell adhesion molecule (EpCAM) $)^{\text {high }} / \mathrm{CD} 44^{+} / \mathrm{CD} 166^{+}$[15]. Identification of $\mathrm{CD} 133^{+} \mathrm{CD} 26^{+} \mathrm{CD} 44^{+}$colorectal $\mathrm{CSC}$ is a relatively recent report. A study of 43 patient tumours (27 without liver metastasis, 5 with synchronous liver metastasis on presentation and 15 metachronous liver metastases) analyzed the expression of these markers in dissociated tumour cells by three-color flow cytometry [16]. The most important finding of this study is the ability of $\mathrm{CD} 26^{+}$cells to cause liver metastasis when injected into the cecum of mice irrespective of CD133 or CD44 expression. However, co-expression of CD133 and CD44 did increase the tumourigenic capacity of $\mathrm{CD} 26^{+}$cells. Transient transfection of CD26 siRNA decreased migratory and invasive capacities of the CD26 ${ }^{+}$ CSCs suggesting that CD26 plays a functional role in the invasive capacity of $\mathrm{CD} 26^{+} \mathrm{CSC}$.

CD44 is overexpressed in colon cancers, which often have hyperactivation of the WNT signaling pathway. Deletion of the WNT target, $\mathrm{CD} 44$, in $\mathrm{Apc}(\mathrm{Min} /+)$ mice attenuated intestinal tumourigenesis [17]. $\mathrm{CD} 44$, a class I transmembrane glycoprotein that binds to hyaluronan, with various roles from adhesion to signaling [18], is known as a marker of tumour aggressiveness playing a functional role in metastasis [19] and is also a promising marker of CSCs [20]. However, CD44 expression is not limited to CSCs, as many non-CSCs can also express CD44. Hence, combination of CD44 with other markers is used to identify potential CSCs [21]. CD44 expression has been associated with poor patient survival in multiple cancer types, including colon cancers [18]. In intestinal stem cells and in transit-amplifying cells distinct CD44 isoforms contribute to tumour formation [22].

CD133 is a five-transmembrane glycoprotein and identified as a marker of neural CSCs and normal primitive cells of the hematopoietic, epithelial and endothelial lineages [23-25]. Interestingly, CD133+ cells from colon tumours have stem-like properties and grow exponentially in vitro as undifferentiated tumour organoids. In colon cancer, they account for approximately $2.5 \%$ of the tumour cells [14] and can initiate tumour growth in immune-deficient mice [26]. Recent findings indicated $\mathrm{CD}_{133^{+}}$cells comprised of both stem-like and differentiated progenitor cells suggesting the $\mathrm{CD}_{133^{+}}$sub-population (with a combination marker) had multi-functional roles. Co-expression of epithelial cell adhesion molecule (EpCam) and $\mathrm{CD} 44^{+}$in $\mathrm{CD} 133^{+}$ fraction further differentiates a sub-group which possess tumourigenic properties [15].

CD26 or Dipeptidyl-peptidase IV (DPPIV) is a serine peptidase involved in cell differentiation, adhesion, immune modulation and apoptosis, all functions which control neoplastic transformation, and its' up-regulation was observed in colorectal tumours [27]. An antimigratory and invasive effect was found with ATP-competitive pan Raf265 inhibitor treatment in combination with 5FU by targeting CD26+ CRC cells [28].

In the present study, we used primary colon tissues to isolate CSC and characterize cultured organoids.

\section{Materials and Methods}

All chemicals were obtained from Sigma-Aldrich unless stated otherwise.

\section{Patient specimen collection}

A total of 22 colon specimen were obtained (following consent) from patients, undergoing screening colonoscopy and/or invasive surgery from 2015-2016, at SKMCH\&RC, Pakistan. Ethical approval of the study was obtained from the Internal Review Board on Human Research at SKMCH\&RC. Specimens of normal colonic mucosa and colon nonadenomatous polyp biopsies $\left(2 \mathrm{~mm}^{3}\right.$ to $5 \mathrm{~mm}^{3}$ ), to be used as noncancer controls, were collected from non-cancer patients undergoing screening colonoscopy. Specimens were collected within 30 minutes of resection and placed in RMPI medium with $5 \mathrm{x}$ antibiotic $(500 \mu \mathrm{g} / \mathrm{mL}$ streptomycin, 500 Units penicillin and $1.25 \mu \mathrm{g} / \mathrm{mL}$ amphotericin-B) immediately. A part of the tissue was fixed in formalin for embedding into paraffin wax (for histo-pathological diagnosis) and the remaining tissue was either processed for isolation of cells or stored at $-80^{\circ} \mathrm{C}$ in $1 \mathrm{x}$ phosphate-buffered saline (PBS). Colon tumour tissues were obtained during laparoscopy or open surgery performed for tumour resection. These specimens were collected and processed as described above. The manifestation of normal, hyperplastic or neoplastic cells in the attained specimen was confirmed by two independent histo-pathologists. The clinic-pathological features of patients are summarized in Table 1.

\section{Single-cell suspension formation from colon tissues}

Mucus containing samples were incubated in sputolysin reagent (100 mM PBS (pH 7.0), $6.5 \mathrm{mM}$ Dithiothreitol) for 15 minutes at room temperature and rinsed with Hank's balanced salt solution (HBSS). A single-cell suspension of each biopsy was generated using the method of Pang et al. [16]. Briefly, fresh specimens were washed with $1 \times$ PBS, minced on ice and transferred to $15 \mathrm{~mL}$ centrifuge tube containing Dulbecco's Modified eagles Medium (DMEM), combination of Hyaluronidase (Calbiochem, Heidelberg, USA) and Collagenase IV (Invitrogen, Paisley, UK) for enzymatic disaggregation. Complete digestion was ensured by stirring the mixture every 15 minutes and incubation at $37^{\circ} \mathrm{C}$ for up to 3 hours. The cellular debris in the resulting single-cell suspension was filtered using $40 \mu \mathrm{m}$ nylon mesh and washed twice with HBSS/2\% Hank's buffered salt solution in heat inactivated calf serum (HICS). Histopaque-1077 centrifugation as per the manufacturer's instructions was used to remove red blood cells.

\section{Magnetic bead affinity cell sorting (MACS) of $\mathrm{CD}^{2}{ }^{+}{ }^{+} \mathrm{CD}^{+}{ }^{+} \mathrm{CD}^{+} 4^{+}$}

From the resulting single-cell suspension, $\mathrm{CD} 133^{+} \mathrm{CD} 26^{+} \mathrm{CD} 44^{+}$ were isolated using a Miltenyi Biotech kit as per the manufacturer's instructions. Briefly, single-cell suspensions were incubated with a monoclonal CD133-APC (Miltenyi Biotech, Bergisch Gladbach, Germany) antibody, labeled with MicroBeads (Miltenyi Biotech) for $30 \mathrm{~min}$ at $4^{\circ} \mathrm{C}$. Anti-APC Multisort Kit (Miltenyi Biotech) was used to obtain the $\mathrm{CD}_{133^{+}}$cells followed by cleavage from the MicroBeads using release reagent from the kit. $\mathrm{CD} 133^{+}$cells were incubated with CD26PE (Miltenyi Biotech) antibody and Anti-PE Multisort Kit (Miltenyi Biotech) was used to obtain $\mathrm{CD} 133^{+} \mathrm{CD} 26^{+}$cells. The microbeads were cleaved from the $\mathrm{CD} 133^{+} \mathrm{CD} 26^{+}$cells by using the release reagent in the Multisort kit. The $\mathrm{CD} 133^{+} \mathrm{CD} 26^{+}$cells were incubated with the CD44 Microbeads (Miltenyi Biotech,) to obtain CD133 ${ }^{+} \mathrm{CD} 26^{+} \mathrm{CD} 44^{+}$cells. The filtrate was collected containing CD44-CD26-CD133 cells. 


\begin{tabular}{|c|c|c|c|c|c|c|c|c|c|c|c|c|c|c|c|c|}
\hline 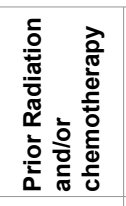 & $\stackrel{\circ}{z}$ & $\stackrel{0}{z}$ & $\stackrel{0}{z}$ & $\stackrel{0}{z}$ & $\stackrel{0}{z}$ & $\stackrel{0}{z}$ & $\stackrel{0}{z}$ & $\stackrel{0}{z}$ & 운 & $\stackrel{0}{z}$ & $\stackrel{0}{z}$ & zo & $\stackrel{0}{z}$ & $\stackrel{2}{z}$ & $\stackrel{\circ}{z}$ & z \\
\hline 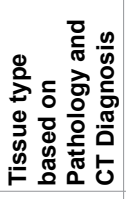 & 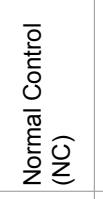 & 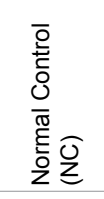 & 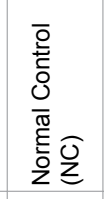 & 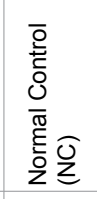 & 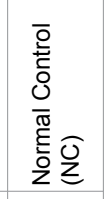 & 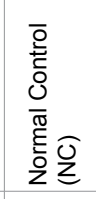 & 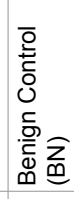 & 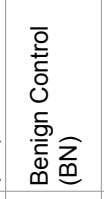 & 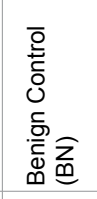 & 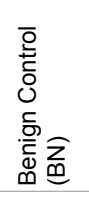 & 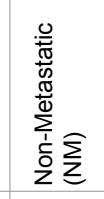 & 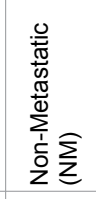 & 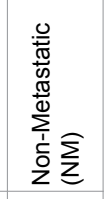 & 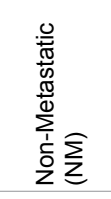 & 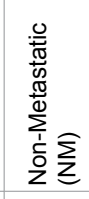 & 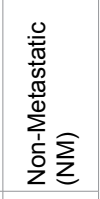 \\
\hline $\begin{array}{l}\frac{0}{0} \\
0 \\
0 \\
\frac{5}{0} \\
\overline{0} \\
5 \\
0\end{array}$ & 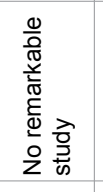 & 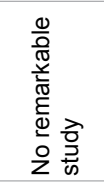 & 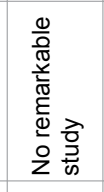 & 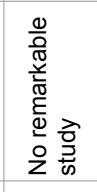 & 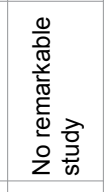 & 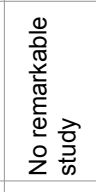 & 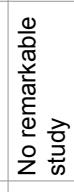 & 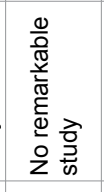 & 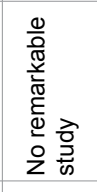 & 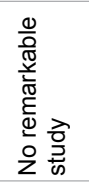 & 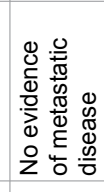 & 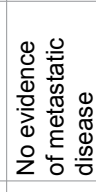 & 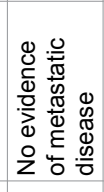 & 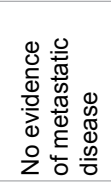 & 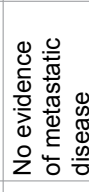 & 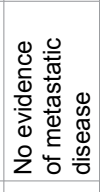 \\
\hline $\begin{array}{l}\frac{0}{0} \\
\frac{0}{50} \\
\overline{0} \\
\frac{0}{0} \\
\frac{0}{0} \\
\frac{0}{I}\end{array}$ & $\Sigma$ & $\Sigma$ & $\Sigma$ & $\Sigma$ & $\mathbb{z}$ & $\mathbb{z}$ & $\Sigma$ & $\Sigma$ & $\mathbb{z}$ & $\Sigma$ & 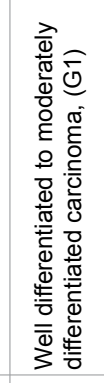 & 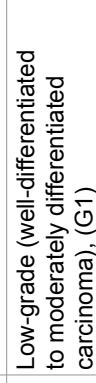 & 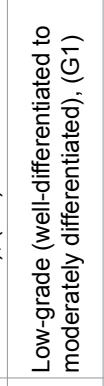 & 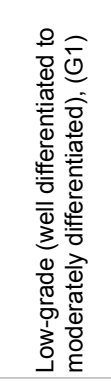 & 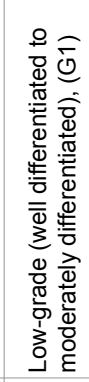 & 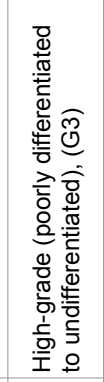 \\
\hline 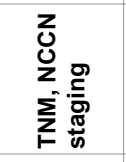 & 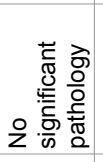 & 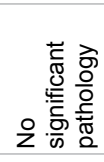 & 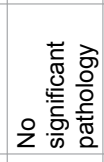 & 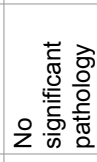 & 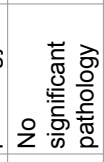 & 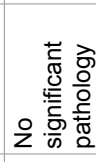 & 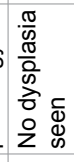 & 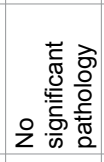 & 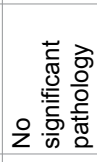 & 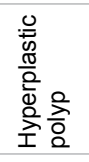 & 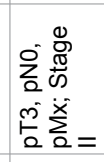 & 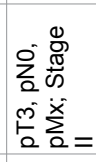 & 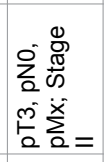 & 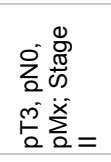 & 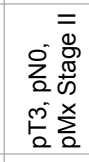 & 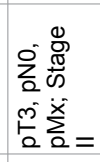 \\
\hline 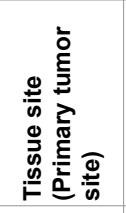 & 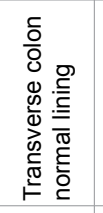 & 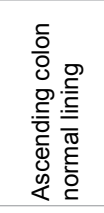 & 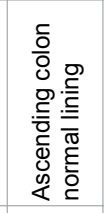 & 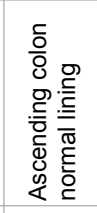 & 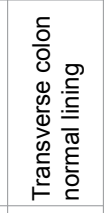 & 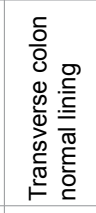 & 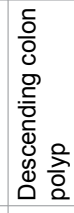 & 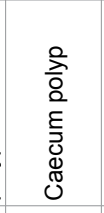 & 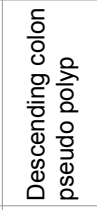 & 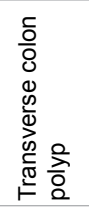 & 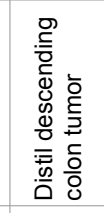 & 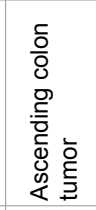 & 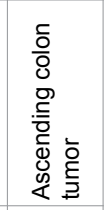 & 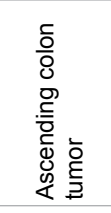 & 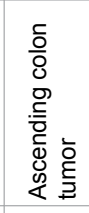 & 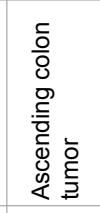 \\
\hline
\end{tabular}

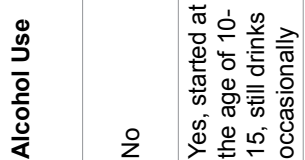

\begin{tabular}{|c|c|c|c|c|c|c|c|c|c|c|c|c|c|c|c|c|}
\hline$\frac{0}{4}$ & z & $\stackrel{0}{\circlearrowright} \stackrel{0}{=}$ & z & 운 & z & zo & z & zo & zo & z & zo & z & zo & 울 & z & zo \\
\hline 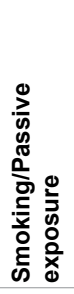 & 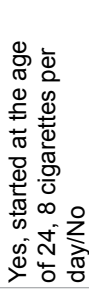 & 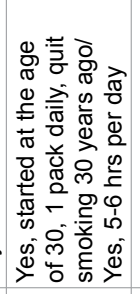 & 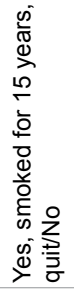 & $\begin{array}{l}\text { ¿o } \\
\text { 2 }\end{array}$ & 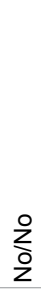 & $\begin{array}{l}\frac{0}{2} \\
\frac{1}{2}\end{array}$ & 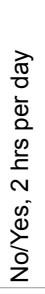 & $\begin{array}{l}\stackrel{0}{0} \\
\text { z }\end{array}$ & $\begin{array}{l}\stackrel{0}{0} \\
\text { L }\end{array}$ & 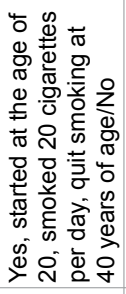 & 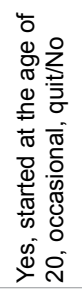 & 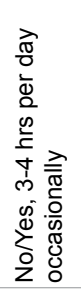 & $\begin{array}{l}\sum_{0} \\
\frac{0}{2}\end{array}$ & 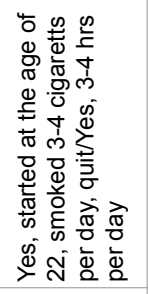 & $\begin{array}{l}\stackrel{0}{2} \\
\frac{0}{2}\end{array}$ & $\begin{array}{l}\stackrel{0}{2} \\
\frac{0}{2}\end{array}$ \\
\hline 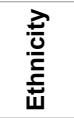 & 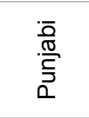 & 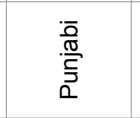 & 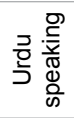 & 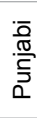 & $\begin{array}{l}\frac{\bar{a}}{\bar{\sigma}} \\
\frac{\bar{s}}{0} \\
0\end{array}$ & 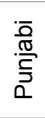 & 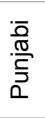 & 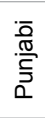 & 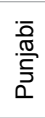 & 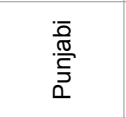 & $\begin{array}{l}\overline{\bar{a}} \\
\overline{\frac{0}{5}} \\
\overline{0} \\
0\end{array}$ & $\begin{array}{l}\overline{\bar{a}} \\
\overline{\frac{0}{5}} \\
\overline{0} \\
0\end{array}$ & 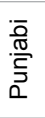 & 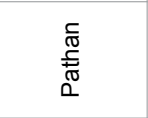 & 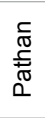 & $\begin{array}{l}\frac{c}{\sigma} \\
\frac{w}{\sigma} \\
0 \\
0\end{array}$ \\
\hline$\stackrel{8}{\&}$ & $\forall$ & 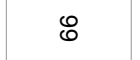 & 2 & $\bar{m}$ & ๕ீ & $\approx$ & in & q & $\stackrel{\infty}{\sim}$ & \& & ஜீ & i̊ & 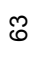 & ర్ల & $\stackrel{\infty}{\circ}$ & \& \\
\hline 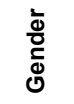 & $\Sigma$ & $\Sigma$ & $\Sigma$ & ч & $\Sigma$ & $\Sigma$ & 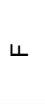 & $\Sigma$ & \llcorner & $\Sigma$ & $\Sigma$ & 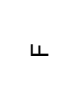 & 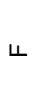 & $\Sigma$ & $\Sigma$ & ч \\
\hline 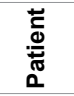 & r & N & $m$ & 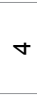 & 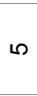 & 0 & r & $\infty$ & $\sigma$ & 우 & $\digamma$ & $\simeq$ & $\stackrel{m}{=}$ & $\stackrel{\Xi}{\check{2}}$ & $\stackrel{6}{\leftarrow}$ & 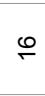 \\
\hline
\end{tabular}


Citation: Tariq S, Tahseen M, Hassan M, Masood MA, Khattak S, et al. (2017) Stem Cell Organoids in Primary Cultures of Human Non-Malignant and Malignant Colon. J Mol Biomark Diagn 8: 346. doi: 10.4172/2155-9929.1000346

Page 4 of 6

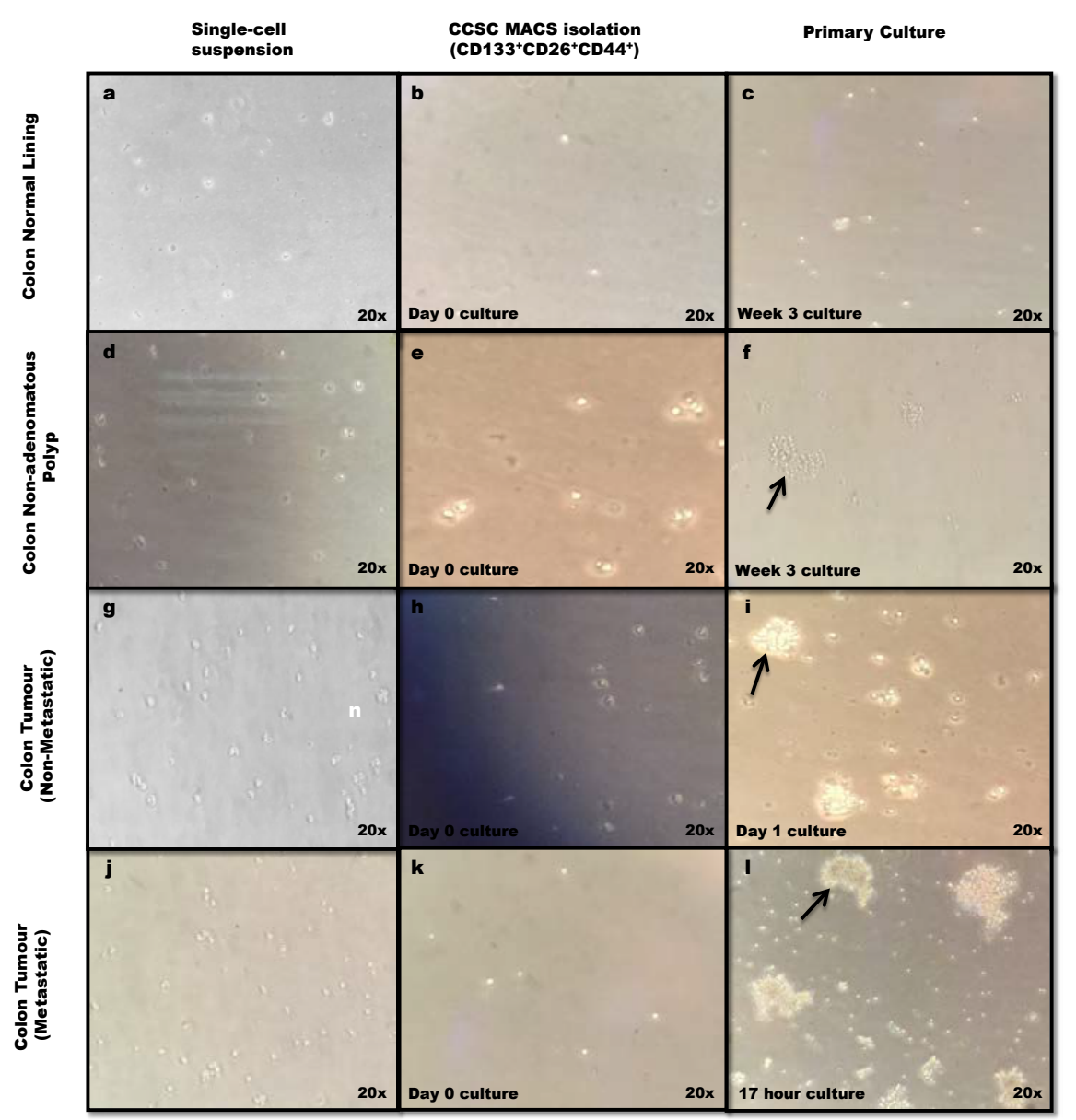

Figure 1: Representative phase contrast images of spheres (arrows) formed in suspension in primary cultures of colon tissues (f, i, l) in growth factor supplemented medium in non-adhesive ultra-low attachment flasks; Panel 1: Colon normal lining (Non-cancer) (a-c): (a) trypsin-digested single-cell suspension, (b) isolated $\mathrm{CD} 133^{+} \mathrm{CD} 26^{+} \mathrm{CD} 44^{+}$cells cultured under stem cell conditions, (c) No spheres formed within 3 weeks and cells started to differentiate onwards. Panel 2: Colon nonadenomatous polyp (Non-cancer) (d-f): (d) trypsin-digested single-cell suspension, (e) isolated CD133+CD26+CD44+ cells cultured under stem cell conditions, (f) created and survived for 3 weeks. One generation of secondary spheres were formed. Panel 3: Colon tumour (Non-Metastatic) (g-i): (g) trypsin-digested singlecell suspension, (h) isolated $\mathrm{CD} 133^{+} \mathrm{CD} 26^{+} \mathrm{CD} 44^{+}$cells cultured under stem cell conditions, (i) created within 24 hours and survived for several. Re-passaged for 3 generations. Panel 4: Colon tumour (Metastatic) (j-I): (j) trypsin-digested single-cell suspension, $(k)$ isolated $C^{2} 133^{+} C D 266^{+} C D 44^{+}$cells cultured under stem cell conditions, (l) created within 24 hours and survived for several. Re-passaged for 5 generations. Magnification 20x.

\section{Primary culture of $\mathrm{CD} 33^{+} \mathrm{CD} 26^{+} \mathrm{CD} 44^{+}$cell organoids}

Separate cultures for each type of fresh specimen (colon normal lining, colon non-adenomatous polyp and colon tumour) were set up.

The resulting single-cells $\left(\mathrm{CD} 133^{+} \mathrm{CD} 26^{+} \mathrm{CD} 44^{+}\right)$were passed through a $40 \mu \mathrm{m}$ filter and placed in stem cell conditions (nonadherent stem cell medium; DMEM/F12, $1 \times$ antibiotic/antimycotic mixture, $0.4 \%$ bovine serum albumin (BSA), $1 \%$ glutamine, insulin 5 $\mu \mathrm{g} / \mathrm{mL}, 20 \mathrm{ng} / \mathrm{mL}$ epidermal growth factor (EGF) (Invitrogen), $10 \mathrm{ng} /$ $\mathrm{mL}$ basic fibroblast growth factor (bFGF) [Invitrogen]) without feeder cells (fibroblasts or amniotic membrane) to test their ability for forming organoids containing CSCs. Non-adherent stem cell medium $(10 \mathrm{~mL})$ containing $1 \times 10^{3}$ cells were cultured in ultra-low attachment $25 \mathrm{~mm}^{2}$ culture flasks (Corning Inc., Corning, NY) (29). The culture flasks were placed at $37^{\circ} \mathrm{C}, 85 \%$ humidity and $5 \% \mathrm{CO}_{2}$ replacing fresh medium once/week. When floating organoids were formed, they were collected by centrifugation. Parallel cultures under adherent condition $(10 \%$ BSA, $1 \%$ glutamine, no EGF and bFGF) were set up in collagen-coated culture flasks. The filtrate containing CD44-CD26-CD133 cells was also cultured both in adherent and non-adherent conditions $(1 \times$ antibiotic/ antimycotic mixture, $10 \%$ bovine serum albumin, $1 \%$ glutamine, no EGF and bFGF) in collagen-coated culture flasks.

\section{Self-renewal assay}

The organoids, collected by centrifugation and digested to singlecell suspension using Dispase II (20 U/mL of DMEM), were filtered through $40 \mu \mathrm{m}$ filter. For daughter organoids formation and colony formation, 250 cells $/ \mathrm{cm}^{2}$ in $10 \mathrm{~mL}$ of medium were cultured in nonadherent or adherent $25 \mathrm{~cm}^{2}$ culture flasks, respectively. The organoids in suspension and adhesive colonies were observed using phase contrast microscope (Labomed TCM 400). Trypan blue exclusion method was used to test the viability of the cells.

\section{Results}

\section{Organoid formation in primary cultures of $\mathrm{CD} 133^{+} \mathrm{CD}^{+}{ }^{+} \mathrm{CD} 44^{+}$Cells}

The single-cell suspension from each type of colon normal lining (Figure 1a), colon non-adenomatous polyp (Figure 1d) and colon tumour (Figure $1 \mathrm{~g}$ and $1 \mathrm{j}$ ) was incubated in non-adherent stem cell 
Citation: Tariq S, Tahseen M, Hassan M, Masood MA, Khattak S, et al. (2017) Stem Cell Organoids in Primary Cultures of Human Non-Malignant and Malignant Colon. J Mol Biomark Diagn 8: 346. doi: 10.4172/2155-9929.1000346

Page 5 of 6

\begin{tabular}{|c|c|c|c|c|c|c|c|c|}
\hline \multirow{2}{*}{ Tissue type } & \multicolumn{8}{|c|}{$\mathrm{CD}{ }^{3} 3^{+} \mathrm{CD}^{26}{ }^{+} \mathrm{CD} 4^{+}$Cells } \\
\hline & & Parentspheres & Passage 1 & Passage 2 & Passage 3 & Passage 4 & Passage 5 & Passage 6 \\
\hline Colon Normal Lining & $\begin{array}{l}\text { Duration } \\
\text { Number } \\
\text { Cells/collection }\end{array}$ & $\begin{array}{l}3 \text { weeks } \\
3-5 \\
4-6\end{array}$ & None & NA & NA & NA & NA & NA \\
\hline Colon Non-Adenomatous Polyp & $\begin{array}{l}\text { Duration } \\
\text { Number } \\
\text { Cells/sphere }\end{array}$ & $\begin{array}{l}3 \text { weeks } \\
10-20 \\
30-60\end{array}$ & $\begin{array}{l}1 \text { week } \\
10-15 \\
10-20\end{array}$ & None & NA & NA & NA & NA \\
\hline $\begin{array}{l}\text { Colon tumour } \\
\text { (Non-Metastatic) }\end{array}$ & $\begin{array}{l}\text { Duration } \\
\text { Number } \\
\text { Cells/sphere }\end{array}$ & $\begin{array}{l}1 \text { day } \\
50-100 \\
50-170\end{array}$ & $\begin{array}{l}1 \text { day } \\
30-70 \\
50-100\end{array}$ & $\begin{array}{l}1 \text { day } \\
20-25 \\
20-50\end{array}$ & $\begin{array}{l}1 \text { day } \\
10-15 \\
10-20\end{array}$ & None & NA & NA \\
\hline Colon tumour (Metastatic) & $\begin{array}{l}\text { Duration } \\
\text { Number } \\
\text { Cells/sphere }\end{array}$ & $\begin{array}{l}<24 \text { hours } \\
50-130 \\
50-170\end{array}$ & $\begin{array}{l}<24 \text { hours } \\
50-100 \\
50-130\end{array}$ & $\begin{array}{l}\leq 24 \text { hours } \\
50-70 \\
30-90\end{array}$ & $\begin{array}{l}1 \text { day } \\
30-40 \\
20-30\end{array}$ & $\begin{array}{l}1 \text { day } \\
30-40 \\
15-25\end{array}$ & $\begin{array}{l}2 \text { days } \\
15-25 \\
\text { Up to } 10\end{array}$ & None \\
\hline
\end{tabular}

Cells plated at a density of $250 / \mathrm{cm}^{2}$ in $10 \mathrm{~mL}$ of conditioned media in Ultra-Low attachment culture flasks

Triplicate analyses were performed from each sample type

NA: Not applicable

Table 2: Number and size of spheres in MACS isolated CD133+CD26+CD44+ cell cultures.

medium to observe their organoid formation patterns in primary cell culture. Purified $\mathrm{CD} 133^{+} \mathrm{CD} 26^{+} \mathrm{CD} 44^{+}$cells exhibited sphere-forming abilities compared to the CD133-CD26 CD44 equivalents. The MACS isolated cells (Figure 1b) from non-cancer colon normal lining did not produce characteristic compact round organoids (Figure 1c). The isolated $\mathrm{CD} 133^{+} \mathrm{CD} 26^{+} \mathrm{CD} 44^{+}$cells (Figure 1e) from colon nonadenomatous polyp showed a slow growth pattern of loosely compact organoids (Figure 1f) which did not survive for more than a month. However, organoids (which mimic tumor in a flask) were generated at much faster rate within 1 day from $\mathrm{CD} 133^{+} \mathrm{CD} 26^{+} \mathrm{CD} 44^{+}$cells isolated from non-metastatic (Stage II) tumours (Figure 1i) organoids, whereas those isolated from advanced disease metastatic (Stage IV) tumours (Figure 1l) exhibited highest growth rate and cell numbers in less than 24 hours. After 24 hours of culture, floating undifferentiated CD44 CD26 ${ }^{-}$D $133^{-}$cells start to adhere to the flask (both collagen coated and non-coated) and attain the shape similar to that of colon epithelial cells.

\section{Self-renewal of cancer cell organoids}

The ability for self-renewal and adoption of a spherical morphology, attributed to the presence of CSCs, were tested by collecting the $\mathrm{CD} 133^{+} \mathrm{CD} 26^{+} \mathrm{CD} 44^{+}$cell organoids and culturing them after dissociation into single-cells. The secondary organoids were formed within 1 week from colon non-adenomatous tissue but did not regenerate secondary organoids on $2^{\text {nd }}$ passage. A progressive loss of regenerative ability by the third passage was observed with primary cells from non-metastatic tumours and by fifth passage for those from metastatic cancers (Table 2).

\section{Discussion}

The aim of our pilot study was to isolate $\mathrm{CD} 133^{+} \mathrm{CD} 26^{+} \mathrm{CD} 44^{+} \mathrm{CSCs}$ from patient colon tumours, culture spheres or organoids and observe their proliferation in primary cultures. We setup primary cultures from human colon tumour to grow cancer cell organoids, isolated $\mathrm{CD} 133^{+} \mathrm{CD} 26^{+} \mathrm{CD} 44^{+}$cells by MACS followed by culturing to produce cell organoids. Normal tissues were processed as the tumor tissues using same method of isolation. Under the same culture conditions, the non-cancer cells did not produce organoids while those from nonmetastatic and metastatic tumours produced compact, round and multi-cellular organoids (Figure 1) confirming that only tumour cells have self-renewal abilities. The non-cancer tissue produced single-cells which died after few weeks of culture. The organoids from different tissue sources, survived over different time periods (1-30 days), which could be attributed to the different type of cells with specific set of mutated genes in various tissues (Table 2).
Comprehensive comparisons of gene pool and proteome of these organoids from various stages of cancer can be helpful in monitoring CRC progression and metastasis. These genetic and proteomic differences between non-adenomatous polyps and normal colon lining, which did not translate into identifiable histologic features, could be the reason why non-adenomatous polyps demonstrated a loosely compact sphere formation pattern (Figure 1f) absent from normal colon lining cultures. This organoid model may provide the means for in-depth 'omics' analyses to understand the dynamics of the cancer.

\section{Conclusion}

In summary, we successfully isolated $\mathrm{CD} 133^{+} \mathrm{CD} 26^{+} \mathrm{CD} 44^{+}$cells from human colon tissues by magnetic beads and generated colon cancer cell organoids in primary cultures in supplemented medium. The potential applications for colon organoids (potentially harboring CSCs) characterization include developing methods for CSC detection, CSC resistance to cancer therapies, identifying new potential therapeutic targets and study mechanism of suppression of cancer metastasis.

\section{Acknowledgment}

We thank Shaukat Khanum Memorial Cancer Hospital and Research Centre, Lahore, Pakistan for funding this research. We thank Dr. Sadaf Mufty and Dr. Afshan Salman for assistance in the collection of tissue biopsies. We are grateful to the patients who agreed to take part in this research.

\section{References}

1. Karanikas M, Esebidis $A(2016)$ Increasing incidence of colon cancer in patients $<50$ years old: A new entity? Ann Transl Med 4: 164.

2. http://gco.iarc.fr/today/home

3. Nelson H, Petrelli N, Carlin A, Couture J, Fleshman J, et al. (2001) Guidelines 2000 for colon and rectal cancer surgery. J Nat Can Inst 93: 583-596.

4. Sancho E, Batlle E, Clevers H (2004) Signaling pathways in intestinal development and cancer. Ann rev cell dev biol 20: 695-723.

5. Siegel RL, Miller KD, Jemal A (2016) Cancer statistics, 2016. CA Cancer J Clin 66: 7-30.

6. Bhurgri Y, Bhurgri A, Nishter S, Ahmed A, Usman A, et al. (2006) Pakistan: Country profile of cancer and cancer control 1995-2004. J Pak Med Assoc 56 124-130.

7. Shaikh AJ, Raza S, Shaikh AA, Idress R, Kumar S, et al. (2009) Demographics pathologic patterns and long-term survival in operable colon cancers: Local experience in Pakistan. Asian Pac J Cancer Prev 10: 361-364.

8. Bonnet D, Dick JE (1997) Human acute myeloid leukemia is organized as a hierarchy that originates from a primitive hematopoietic cell. Nat med 3 . 730-737.

9. Al-Hajj M, Wicha MS, Benito-Hernandez A, Morrison SJ, Clarke MF (2003) 
Citation: Tariq S, Tahseen M, Hassan M, Masood MA, Khattak S, et al. (2017) Stem Cell Organoids in Primary Cultures of Human Non-Malignant and Malignant Colon. J Mol Biomark Diagn 8: 346. doi: 10.4172/2155-9929.1000346

Page 6 of 6

Prospective identification of tumorigenic breast cancer cells. Proc Natl Acad Sci USA 100: 3983-3988.

10. Ponti D, Costa A, Zaffaroni N, Pratesi G, Petrangolini G, et al. (2005) Isolation and in vitro propagation of tumorigenic breast cancer cells with stem/progenitor cell properties. Cancer res 65: 5506-5511.

11. Singh SK, Clarke ID, Terasaki M, Bonn VE, Hawkins C, et al. (2003) Identification of a cancer stem cell in human brain tumors. Cancer Res 63: 5821-5828.

12. Patrawala L, Calhoun T, Schneider-Broussard R, Li H, Bhatia B, et al. (2006) Highly purified CD44+ prostate cancer cells from xenograft human tumors are enriched in tumorigenic and metastatic progenitor cells. Oncogene 25: 1696-1708.

13. Li C, Heidt DG, Dalerba P, Burant CF, Zhang L, et al. (2007) Identification of pancreatic cancer stem cells. Cancer Res 67: 1030-1037.

14. Ricci-Vitiani L, Lombardi D, Pilozzi E, Biffoni M, Todaro M, et al. (2007) Identification and expansion of human colon-cancer-initiating cells. Nature 445 : 111-115.

15. Dalerba P, Dylla SJ, Park I-K, Liu R, Wang X, Cho RW, et al. (2007) Phenotypic characterization of human colorectal cancer stem cells. PNAS 104: 10158-10163.

16. Pang R, Law WL, Chu ACY, Poon JT, Lam CSC, et al. (2010) A subpopulation of CD26+ cancer stem cells with metastatic capacity in human colorecta cancer. Cell stem cell 6: 603-615.

17. Zeilstra J, Joosten SP, Dokter M, Verwiel E, Spaargaren M, et al. (2008) Deletion of the WNT target and cancer stem cell marker CD44 in Apc(Min/+) mice attenuates intestinal tumorigenesis. Cancer Res 68: 3655-3661.

18. Ponta H, Sherman L, Herrlich PA (2003) CD44: From adhesion molecules to signalling regulators. Nat Rev Mol Cell Biol 4: 33-45.

19. Joshua B, Kaplan MJ, Doweck I, Pai R, Weissman IL, et al. (2012) Frequency of cells expressing CD44, a Head and Neck cancer stem cell marker: Correlation with tumor aggressiveness. Head Neck 34: 42-49.

20. Jaggupilli A (2012) Significance of CD44 and CD24 as cancer stem cell markers: An enduring ambiguity. Clin Dev Immunol 2012: 11.

21. Zöller M (2011) CD44: Can a cancer-initiating cell profit from an abundantly expressed molecule? Nat Rev Cancer 11: 254-267.

22. Guo W, Frenette PS (2014) Alternative CD44 splicing in intestinal stem cells and tumorigenesis. Oncogene 33: 537-538.

23. Salven P, Mustjoki S, Alitalo R, Alitalo K, Rafii S (2003) VEGFR-3 and CD133 identify a population of CD34+ lymphatic/vascular endothelial precursor cells. Blood 101: 168-172.

24. Uchida N, Buck DW, He D, Reitsma MJ, Masek M, et al. (2000) Direct isolation of human central nervous system stem cells. PNAS 97: 14720-14725.

25. Yin AH, Miraglia S, Zanjani ED, Almeida-Porada G, Ogawa M, et al. (1997) AC133, a novel marker for human hematopoietic stem and progenitor cells. Blood 90: 5002-5012.

26. O'Brien CA, Pollett A, Gallinger S, Dick JE (2007) A human colon cancer cell capable of initiating tumour growth in immunodeficient mice. Nature 445: 106-110.

27. Larrinaga G, Perez I, Sanz B, Beitia M, Errarte P, et al. (2015) Dipeptidylpeptidase IV activity is correlated with colorectal cancer prognosis. PLoS One 10: e0119436.

28. Chow AK, Cheng NS, Lam CS, Ng L, Wong SK, et al. (2015) Preclinical analysis of the anti-tumor and anti-metastatic effects of Raf265 on colon cancer cells and CD26(+) cancer stem cells in colorectal carcinoma. Mol cancer 14: 80.

29. Ponti D, Costa A, Zaffaroni N, Pratesi G, Petrangolini G, et al. (2005) Isolation and in vitro propagation of tumorigenic breast cancer cells with stem/progenitor cell properties. Cancer Res 65: 5506-5511. 